\title{
A Novel Approach to the Detection and Characterization of PAH Cations and PAH-Photoproducts
}

\author{
D. L. Kokkin ${ }^{1}$, A. Simon ${ }^{2}$, C. Marshall ${ }^{1}$, A. Bonnamy ${ }^{1}$ and C. Joblin ${ }^{1}$ \\ ${ }^{1}$ IRAP, Université de Toulouse (UPS-OMP) and CNRS; 9 Av. du Colonel Roche, BP 44346, \\ 31028 Toulouse Cedex 4, France \\ email: Damian.Kokkin@irap.omp.eu \\ ${ }^{2}$ LCPQ, Université de Toulouse (UPS-IRSAMC) and CNRS; 118 Route de Narbonne, 31062 \\ Toulouse Cedex 09, France
}

\begin{abstract}
Cationic polycyclic aromatic hydrocarbons (PAHs) are attractive candidates for the Diffuse Interstellar Bands, but to date not a single PAH species has been identified on the basis of a spectral agreement. This indicates either that the molecular diversity is very large or that the candidates that have been considered are not the correct ones. In particular, small/medium-sized PAH cations are submitted to photodissociation under UV photons from stars. Therefore it is of interest to characterize the spectroscopic properties of key breakdown products. Furthermore, these studies should be performed under conditions that mimic those found in interstellar space, which leads to additional experimental difficulties. We describe the approach we are developing with the PIRENEA set-up and present results on the 1-Methylpyrene cation and photo-derived species. Experimental measurements are guided by calculations based on density functional theory.
\end{abstract}

Keywords. astrochemistry, methods: laboratory, techniques: spectroscopic, ISM: molecules

\section{Introduction}

None of the now 400 plus Diffuse Interstellar Bands (DIBs) observed in the visiblenear infrared region (400-1400 nm) towards reddened stars have been accounted for. The DIBs have been shown to be sensitive to the local physical environment such as UV flux and chemical composition (Salama et al. 1996; Vuong \& Foing 2000; Szczepanski et al. 2002; Cox \& Spaans 2006; Cox et al. 2007). Furthermore, some bands show what maybe rotational or isotopic structure leading to the assertion that the bands are molecular in nature rather then atomic (Galazutdinov et al. 2008; Sarre 2006). Cationic polycyclic aromatic hydrocarbons (PAHs) have been proposed to contribute to the carriers of the DIBs. The PAH hypothesis was first proposed in 1984-85 by Léger et al. and Allamandola and co-workers (Léger \& Puget 1984; Léger \& d'Hendecourt 1985; Crawford et al. 1985; van der Zwet \& Allamandola 1985). Supporting the argument that PAHs are ubiquitous throughout the interstellar medium (ISM) is a series of infrared bands seen in emission and called the aromatic infrared bands or AIBs (see Joblin \& Tielens 2011 for a recent compilation of articles on this subject).

Open shell cationic PAHs have been shown to have strong transitions within the visible and near infrared region unlike their closed shell neutral counterparts and are therefore good candidates for the DIBs (Bréchignac et al. 2001). The challenge to those undertaking laboratory experiments to investigate this hypothesis is to produce PAH cations in an environment comparable to that of the ISM i.e. cold, gas phase, low pressure and measure their electronic spectra. One common technique that is used to isolate cold PAH 


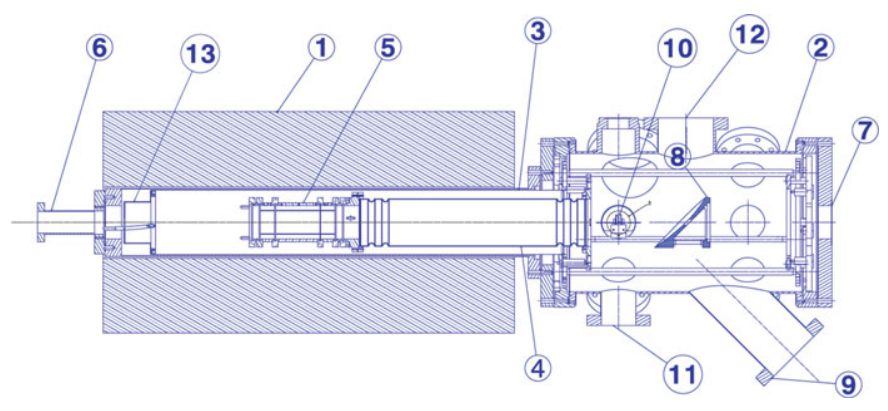

Figure 1. The PIRENEA set-up with (1) the superconducting magnet (5T), (2) the ultra high vacuum vessel, (3) the external cryogenic shield, (4) the inner cryogenic shields, (5) the ICR cell, (6) chemical interface and laser entrance window, (7) secondary laser entrance window, (8) the mirror to focus the light at the center of the trap, (9) turbomolecular pump, (10) sample holder, (11) electronic interface, (12) Xe lamp interface, and (13) baffle.

molecules for spectroscopic purposes is to seed them into an inert matrix such as neon or argon (Halasinski et al. 2005). There are problems associated with this technique, those being: the spectra measured can not be directly compared to those observed, due to the solid state nature of the experiment shifting the observed bands from the pure free molecule position; and additional species other than the cations can be formed upon UV irradiation of the neutral sample such as neutral radicals.

In this proceeding we describe the novel experimental approach that we are developing at IRAP Toulouse, in particular the adaptation of FTICR-MS (Fourier Transform Ion Cyclotron Resonance Mass Spectroscopy) to undertake resonant two color multi-photon dissociation spectroscopy. We present results on the initial breakdown and spectroscopy of the 1-Methylpyrene (1-MP) cation and propose the carriers of the breakdown products, in addition to discussing the implementation of a 2-color multi-photon dissociation scheme.

\section{Experimental Method}

The science and experimental techniques of FTICR-MS including multi-photon dissociation (MPD) spectroscopy have been described and discussed in detail in a number of reviews and articles in relation with the measurement of mass spectra and applying this technique to obtain spectral and structural information (Marshall et al. 1998; Oomens et al. 2006). The PIRENEA setup (Figure 1; "Piège à Ions pour la Recherche et l'Etude de Nouvelles Espèces Astrochimiques") is a home-built FTICR-MS with the additional characteristics of a cold environment, generated by a set of cryogenic shields, that are of interest to approach the physical conditions of the ISM with typical dust temperatures of $10-50 \mathrm{~K}$. It was conceived to perform photo-physical and chemical studies on large molecules and nano-sized particles of astrophysical interest. The trap was designed to study ions with mass-over-charge ratios from 12 up to a few $10000 \mathrm{~m} / \mathrm{z}$, where the ions of interest can be isolated so that interrogation or manipulation can occur. The FTICRMS technique has many advantages. It provides a very high mass-resolution (more than $10^{4}$ ). It allows for a non-destructive mass analysis of the trapped ions making it therefore particularly suitable for photo-physical and MS/MS studies for example investigating breakdown mechanisms and reaction chemistry.

In the present work to produce a target sample of the initial precursor of interest, in this particular case 1-Methylpyrene (Sigma-Aldrich), a few tens of milligrams were powdered, mixed with toluene and then deposited on a sample holder. The sample was subsequently introduced into the PIRENEA setup via a vacuum interlock, after allowing 
for the toluene to evaporate in ambient conditions. Gas phase ions were produced by a two laser desorption-ionization type experiment, where $532 \mathrm{~nm}$ is used to desorb the solid sample and $266 \mathrm{~nm}$ radiation is used to subsequently ionize the free molecules. It is possible to tune the wavelength of the former laser to 266, 355 or $1064 \mathrm{~nm}$, or more selective desorption can be undertaken with implementing an available infrared OPO system. This is one improvement implemented recently compared to the system used in the past (Useli-Bacchitta et al. 2010), resulting in an increase in the stability and reproducibility of the ion population produced.

Some of the produced ions reach the trap, which is closed after the cations have en-

tered. Once there different masses can be isolated by essentially "kicking out" the other masses, such as different isotopologues or fragments, by applying a frequency signal that excite resonantly their cyclotron motion. The trapped mass selected ions are then cooled collisionally with a helium buffer gas that is introduced into the experiment through a tube in contact with the inner cryogenic shield $(\sim 30 \mathrm{~K})$. Following cooling the cations can then be probed spectroscopically by a Nd:YAG pumped OPO laser system or broken further by use of either a Xenon lamp or pulses from another Nd:YAG and the fragments can be subsequently isolated, cooled and probed.

\section{Theoretical Methodology}

To help guide the search for the electronic spectrum of the cations of interest and to understand breakdown schemes further it is necessary to undertake complementary theoretical investigations. The ground state equilibrium structures of the cations of interest were calculated within the density functional theory (DFT) framework, using the B3LYP hybrid functional (Stephens et al. 1994) and the 6-31G(d,p) basis set (Hehre et al. 1972; Hariharan \& Pople 1973). Harmonic infrared spectra were computed for these geometries by diagonalising the mass weighted Hessian matrix. The electronic spectra of the cations were then obtained from time dependent density functional theory (TD-DFT) calculations. Calculations were performed with the Gaussian 09 quantum chemical package (Frisch et al. 2009). Vertical energies were computed with the BLYP functional and the 6-31G(d,p) basis set for the B3LYP/6-31G(d,p) optimised geometries. This approach was previously benchmarked by Sarre and co-workers (Hammonds et al. 2009).

\section{Results and Discussion}

\subsection{Photo-breakdown and spectroscopy of 1-Methylpyrene Cation and its photoproducts}

Léger and co-workers reported on the 1-MP cation electronic spectrum motivated by the close match seen in the matrix spectrum of pyrene cation to the strongest DIB at $4428 \AA$ (Léger et al. 1995). In the experiment, the neutral 1-MP was seeded into a neon matrix and subjected to UV photons to ionize the seeded molecule. The resulting spectrum had a series of bands observed in the 400-850 $\mathrm{nm}$ region including four which were in close agreement in wavelength to known DIBs. This led to the proposition that 1-MP cation and/or its photo-products were possible carriers of these four bands including the DIB at $4428 \AA$.

The 1-MP cation was isolated in PIRENEA so that an isotopic pure sample of cations was present in the trap $(\mathrm{m} / \mathrm{z}=216)$. The trapped ions were then subjected to radiation from a Xenon arc lamp for various irradiation times. Figure 2 i.) shows the observed mass spectrum after trapped 1-MP is subjected to radiation for $0.5 \mathrm{~s}$ and in 2 ii.) for $5 \mathrm{~s}$. It can be seen from these mass spectra that as the irradiation time is increased the level of parent mass signal decreases dramatically with the appearance of peaks at masses $\mathrm{m} / \mathrm{z}$ 

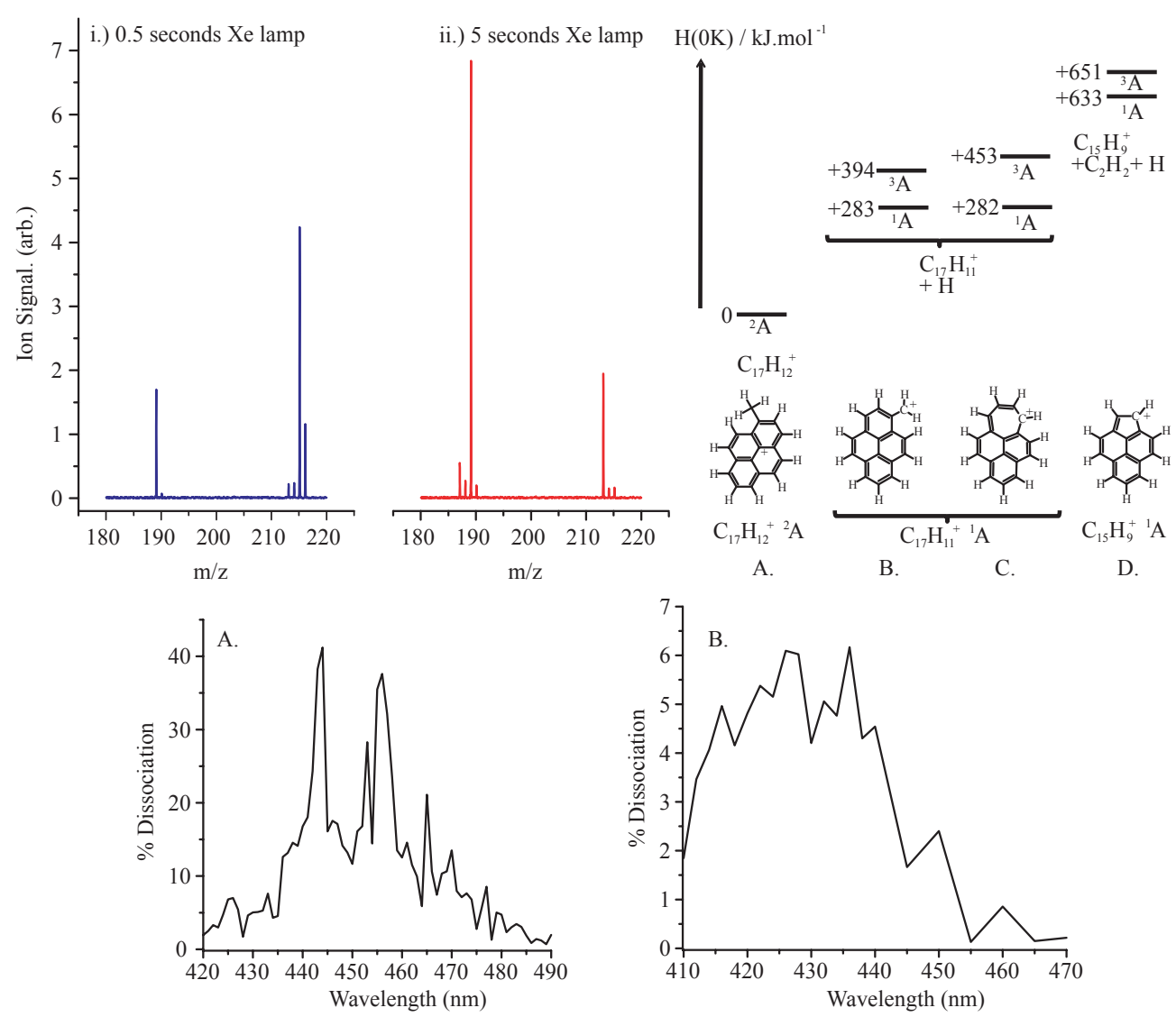

Figure 2. Left: i.) Recorded mass spectrum after the 1-Methylpyrene cation is irradiated with the Xe lamp for $0.5 \mathrm{~s}$ and in ii.) for $5 \mathrm{~s}$. Right: Proposed initial stages in the breakdown scheme (B3LYP/6-31G(d) calculations) with the MPD recorded spectra of A and B (bottom).

$=215$ and 189 primarily. The initial steps in the photo-breakdown of 1-MP cation (A in Figure 2) can then be thought of as rapid $\mathrm{H}$ loss to form $\mathrm{m} / \mathrm{z}=215$ followed by $\mathrm{C}_{2} \mathrm{H}_{2}$ loss forming $\mathrm{m} / \mathrm{z}=189$. A breakdown scheme equivalent to that published for toluene cation is proposed to occur here (Choe 2006; Bullins et al. 2009; Müller et al. 2000). That is after $\mathrm{H}$ loss a rearrangement occurs from 1-Pyrenemethylium (1-PM) cation (B) to a system containing a seven member ring (tropylium like pyrene system) (C). From this intermediate further $\mathrm{C}_{2} \mathrm{H}_{2}$ loss can occur forming a $\mathrm{PAH}$ cation containing a 5 member ring (D). The energetics of this breakdown scheme are given relative to the 1-MP cation parent. As can be seen on Figure 2 (top right), the lowest energy structures of all intermediates were found to be closed shells with all triplet spin-states lying higher in energy.

To confirm the proposed breakdown scheme, spectroscopy has been undertaken using a 1 color-MPD scheme and is presented in the lower part of Figure 2 for 1-MP cation and 1-PM. In MPD, the heating of the cation of interest by each successive photon absorption is expected to broaden the band profile due to anharmonic effects, which is an inherent draw back of 1-color MPD. This is especially the case for 1-PM where multiple carbon-carbon bonds are being dissociated. Still from the bands we measured we can assign the bands seen in the matrix at 4440 and $4560 \AA$ to 1 -MP cation (computed excitation energies and oscillator strengths of 4304 (0.2334) and $4979 \AA$ (0.0185) found with 
TD-DFT) while the band at $4180 \AA$ can be assigned to 1-PM cation (TD-DFT excitation energy and oscillator strength of $4247.1 \AA$ and 0.1520 respectively). Experiments are now underway on the coronene $\left(\mathrm{C}_{24} \mathrm{H}_{12}\right)$ cation, a system with a higher dissociation energy ( $\sim 9 \mathrm{eV}$ in our experimental conditions), to implement a 2-color MPD scheme like that applied successfully by J. Maier and co-workers for the study of cationic carbon chains, with initial results showing a greatly reduced observed line width (Maier et al. 2011).

\section{Acknowledgements}

This work was supported by the French National Program "Physique et Chimie du Milieu Interstellaire" (PCMI) and by French ANR funding with reference ANR-10-BLAN0501-GASPARIM.

\section{References}

Bréchignac, P., Pino, T., \& Boudin, N. 2001, Spectrochimica Acta Part A: Molecular and Biomolecular Spectroscopy, 57, 4, 745-756

Bullins, K. W., Huang, T. T. S., \& Kirkby, S. J. 2009, Int. J. QChem., 109, 1322-1327

Choe, J. C. 2006, J. Phys. Chem. A, 110, 7655-7662

Cox, N. L. J. \& Spaans, M. 2006, A\&A, 451, 3, 973-980

Cox, N. L. J., Cordiner, M. A., Ehrenfreund, P., Kaper, L., Sarre, P. J., Foing, B. H., Spaans, M., Cami, J., Sofia, U. J., Clayton, G. C., Gordon, K. D., \& Salama, F. 2007, A\&AA, 470, $941-955$

Crawford, M. K., Tielens, A. G. G. M., \& Allamandola, L. J. 1985, ApJ, 293, 1, L45-L48

Frisch, M. J., Trucks, G. W., Schlegel, H. B., Scuseria, G. E., Robb, M. A., et al. 2009, Gaussian09 Revision A.1, Gaussian Inc. Wallingford CT

Galazutdinov, G. A., Lo Curto, G., \& Krełowski, J. 2008, MNRAS, 386, 2003-2008

Halasinski, T. M., Salama, F., \& Allamandola, L. J. 2005, ApJ, 628, 555-566

Hammonds, M., Pathak, A., \& Sarre, P. J. 1997, Phys. Chem. Chem. Phys., 22, 11, 4458-4464

Hariharan, P. C. \& Pople, J. A. 1973, Theoretical Chemistry Accounts: Theory, Computation, and Modeling (Theoretica Chimica Acta), 28, 3, 213-222

Hehre, W. J., Ditchfield, R., \& Pople, J. A. 1972, J. Chem. Phys., 56, 5, 2257-2261

Joblin C. \& Tielens A. G. G. M. (eds.) 2011,PAHs and the Universe: A Symposium to Celebrate the 25th Anniversary of the PAH Hypothesis, EAS Pub. Series, Vol. 46

Léger, A. \& Puget, J. L. 1984, A\&A A, 137, 1, L5-L8

Léger, A. \& d'Hendecourt, L. 1985, A\&ऽA, 146, 1, 81-85

Léger, A, d'Hendecourt, L., \& Défourneau 1995, A\& A, 293, 2, L53-L56

Maier, J. P., Chakrabarty, S., Mazzotti, F. J., Rice, C. A., Dietsche, R., Walker G. A. H., \& Bohlender, D. A. 2011, ApJ Letters, 729, L20

Marshall, A. G., Hendrickson, C. L., \& Jackson G. S. 1998, Mass Spec. Rev., 17, 1-35

Müller, A. M., Uiterwaal, C. J. G. J., Witzel, B., Wanner, J., \& Kompa, K.-L. 1995, J. Chem. Phys., 112, 21, 9289-9300

Oomens, J., Sartakov, B. G., Meijer, G., \& von Helden, G. 2006, J. Mass Spec., 254, 1-19

Salama, F., Bakes, E. L. O., Allamandola, L. J., \& Tielens, A. G. G. M. 1996, ApJ, 458, 2, 621-636

Sarre, P. J. 2006, J. Mol. Spec, 238, 1-10

Stephens, P. J., Devlin, F. J., Chabalowski, C. F., \& Frisch, M. J. 1994, J. Phys. Chem.,98, 45, 11623-11627

Szczepanski, J., Banisaukas, J., Vala, M., Hirata, S., \& Wiley, W. R. 2002, J. Phys. Chem. A, 106, 30, 6935-6940

Useli-Bacchitta, F., Bonnamy, A., Mulas, G., Malloci, G., Toublanc, D., \& Joblin, C. 2010, Chem. Phys., 371, 1-3, 16-23

van der Zwet, G. P. \& Allamandola, L. J. 1985, A\& A, 146, 1, 76-80

Vuong, M. H. \& Foing, B. H. 2000, A\&A, 363, L5-L8 